\title{
Cloning and Site-specific Mutagenesis of DS Enzyme Gene of Corynebacterium glutamicum
}

\author{
Hansong $\mathrm{Yu}^{1, \text { a }}$, Shuang $\mathrm{Qu}^{1, \mathrm{a}}$, Yuhua Wang $^{1, \text { a }}$, Chunhong Piao ${ }^{1, \text { a }}$, Junmei \\ $\mathrm{Liu}^{1, \mathrm{a}}$, Yaohui Hu $\mathrm{H}^{1, \mathrm{~b}}$ \\ ${ }^{1}$ College of Food Science and Engineering, JiLin Agriclutural University, \\ Changchun,130118, China \\ aemail: yuhansong@163.com, bemail:huyaohui@vip.163.com
}

Keywords: Fermentation Engineering; Corynebacterium glutamicum; key enzyme of amino acid

\begin{abstract}
In order to obtain the higher activity DS enzyme through genetic engineering technology. The point mutation method is used to get a higher activity recombinant DS enzyme from Corynebacterium glutamicum. At last the point 183 was mutated from L to Q in LG-322.The mutation type is a fundermental of construct a genetic engineering which has high activity DS enzyme.
\end{abstract}

\section{Introduction}

DAHP synthase (DS enzyme) was the first key enzyme that was metabolized to aromatic amino acid [1]. With Arol as the encoding gene, DAHP synthase catalyzed phosphoenolpyruvate (PEP) and erythrose-4-phosphate (E4P) to synthesize 3-deoxy-D-arabino-heptulosonate 7-phosphate (DAHP) and chorismate, which was eventually transformed to tryptophan with joint participation of tryptophan operon and other enzymes and then secreted outside of cells. With the rapid development of molecular biological technology the combination of directed evolution of proteins and gene cloning and expressing methods enabled the allelic substitution of mutant genes of DS enzyme with increased catalytic activity into the genome or their overexpressions in the engineering bacteria [2], so that DS enzyme was metabolized to the direction conducive to tryptophan synthesis to increase tryptophan yield.

In recent years, DS enzyme in E. coli has been deeply studied, whose crystal structure has been submitted to the National Center for Biotechnology Information (NCBI) Structure Database. The site-specific mutagenesis was conducted on His180 by David L et al and on Cys61 and Cys328 by Craig $\mathrm{M}$ et al, respectively, but without improved catalytic activities [3]. Hu Changyun et al in Fudan University confirmed that the enzymatic activities of AroGL175Q, AroGL175A, AroGL175D and Phe209Ser mutants were increased by 1.33, 1.57, 1.65 and 1.8 times. However, there were few reports about DAHP synthase in Corynebacterium glutamicum, the main strain for industrial production of amino acids. Only Liao H-F et al reported that Ser187 had an important allosteric effect on this enzyme. The 150-189 amino acids in AroG gene of DAHP synthase in E. coli were highly conserved with the 158-197 amino acids in Aro I gene of DAHP synthase in Corynebacterium glutamicum, inferring that the catalytic activity of L183Q mutant of DAHP synthase in Corynebacterium glutamicum to this enzyme could also be increased. Therefore, site-directed mutagenesis was used in this study to obtain the gene of DS enzyme by using the genetic engineering method, in order to further enhance the enzymatic activity of DS enzyme, laying the foundation for the metabolic flux regulation in synthesis of amino acids and genetic engineering of high amino acid-producing strains.

\section{Materials and Methods}

\subsection{Materials}

Corynebacterium glutamicum LG-332 and E. coli DH5 $\alpha$ were provided by Biochemical Engineering Laboratory in Food Science and Engineering College of Jilin Agricultural University. 


\subsection{Methods}

\subsubsection{Cloning of DS enzyme in Corynebacterium glutamicum}

2.2.1.1 Primer design and synthesis of DS enzyme in Corynebacterium glutamicum

The specific primers were designed by analysis of the nucleotide sequence (GeneID: 1018979) of DAHP synthase gene (aro I) in Corynebacterium glutamicum ATCC13032 published by GenBank and the multiple cloning site of prokaryotic expression vector pET-28a $(+)$. The upstream primer P1 was 5'-TTTCATATGAGTTCTCCAGTCTCACTCGAAA-3' and the downstream primer P2 was 5'-TTTCTCGAGTTACTTGGCTGCTGCTCGGC-3'. The underlined parts were the restriction sites of Nde I and Xho I. The primers were made by Shanghai Biological Technology Co., Ltd.

2.2.1.2 Extraction of genomic DNA in Corynebacterium glutamicum LG-332

Refer to the Molecular Cloning: A Laboratory Manual, Third Edition [4].

\subsubsection{PCR amplification of DAHP synthase from Corynebacterium glutamicum}

The genomic DNA of Corynebacterium glutamicum as the template, touchdown PCR amplification was conducted according to the reaction system in Table 1 by using P1 and P2 as the primers. The hot-start PCR method was used for reaction conditions as follows: denaturation at 95C for $4 \mathrm{~min}$, adding pause into Primer starHS enzyme at $80 \mathrm{C}$, followed by $94 \mathrm{C} 30 \mathrm{~s}, 60 \mathrm{C}$ 40s and $72 \mathrm{C}$ 120s for two cycles; 94C 30s, 56C 40s and 72C 120s for two cycles; 94C 30s, 56C 40s and 72C 120s for two cycles; 94C 30s, 54C 40s and 72C 120s for two cycles; 94C 30s, 52C 40s and 72C 120s for two cycles; 94C 30s, 50C 40s and 72C 120s for twenty cycles; extension at 72C for $20 \mathrm{~min}$. $0.5 \mu \mathrm{lEx}$ Taq enzyme was added for ligation of A tail. After the reaction, $5 \mu l$ of amplified product was taken for Agarose gel electrophoresis (1\%).

Table 1 The system of PCR

\begin{tabular}{|c|c|}
\hline Compositions & Volume $(\mu 1)$ \\
\hline Distilled water & 29.5 \\
\hline $5 \times$ Primer starHS Buffer & 10.0 \\
\hline dNTP Mixture ( 10mmol/l each) & 4.0 \\
\hline $\mathrm{P} 1(10 \mathrm{pmol} / \mu \mathrm{l})$ & 2.0 \\
\hline $\mathrm{P} 2(10 \mathrm{pmol} / \mu \mathrm{l})$ & 2.0 \\
\hline Genomic DNA & 2.0 \\
\hline Primer starHS $(5 \mathrm{U} / \mu \mathrm{l})$ & 0.5 \\
\hline Total & 50.0 \\
\hline
\end{tabular}

The ordinary operations such as recovery of PCR product, ligation to the vector, extraction of plasmid and transformation referred to the Molecular Cloning: A Laboratory Manual, Third Edition ${ }^{[4]}$.

\subsubsection{Site-directed mutagenesis of DS enzyme in Corynebacterium glutamicum}

2.2.2.1 Selection of mutation sites of DAHP synthase

As shown in Figure 1, the 150-189 amino acids in AroG gene of DAHP synthase in E. coli were highly conserved with the 158-197 amino acids in Aro I gene of DAHP synthase in Corynebacterium glutamicum. Studies have shown that the mutation site in E. coli could increase the catalytic activity of DS enzyme, inferring that L183Q DS mutant of DS enzyme in Corynebacterium glutamicum would increase its catalytic activity.

$\begin{array}{lll}\text { aroG } & \text { PQYI ADLMSWGAIGARTTESQVHRELASGISCPVGFKNGT } & 189 \\ \text { aro1 } & \text { PQYYADTVAWGAIGARTTESQVHRQLASGMSMPIGEKNGT } & 197 \\ \text { Consensus } & \text { PqY ad wgaigarttesqvhr lasg s p gfkngt }\end{array}$

Fig.1 Multiple alignment of the amino-acid sequences of AroG and Aro I

\subsubsection{Design point mutation primers for DAHP synthase}

Two reverse and complementary mutagenic primers were designed according to the sites to be mutated. M1: 5'-TCAGGTGCACCGCCAGCAGGCTTCTGGGATGTCTA-3'; M2: 5'-TAGACATCCCAGAAGCCTGCT GGCGGTGCACCTGA-3'. The underlined parts were the mutation sites. 


\subsubsection{PCR amplification of DAHP mutant genes}

The recombinant plasmid pMD18T-simple-Aro I with correct sequence was used as the template for high-fidelity PCR amplification cycle by using the mutagenic primers. The hot-start PCR method was used for reaction conditions as follows: denaturation at 95C for 4min, adding pause into Primer starHS enzyme at 80C, followed by 94C 30s, 55C 1min and 68C 8min for 35 cycles extension at $72 \mathrm{C}$ for $20 \mathrm{~min}$. After the reaction, $5 \mu \mathrm{l}$ of amplified product was taken for agarose gel electrophoresis (1\%).

2.2.2.4 Primary template of Dpn I enzyme digestion

Dpn I enzyme was directly added into PCR products according to the following system shown in Table 2 at $37 \mathrm{C}$ for $4 \mathrm{~h}$ to complete the digestive process of original template.

Table2. The system of Dpn I digestion

\begin{tabular}{cc}
\hline Compositions & Volume $(\mu 1)$ \\
\hline $\mathrm{ddH}_{2} \mathrm{O}$ & 4.0 \\
$4 \times$ Dpn I buffer & 5.0 \\
Recombinant plasmid & 10.0 \\
Dpn I & 1.0 \\
Total & 20.0
\end{tabular}

The notched digestion products were directly transformed into E. coli DH5 $\alpha$. The positive clones were screened by using ampicillin-resistance $(100 \mathrm{mg} / \mathrm{L})$ and blue-white selection. The routine methods such as identification of the recombinant plasmid and extraction of plasmids referred to the Molecular Cloning: A Laboratory Manual, Third Edition ${ }^{[4]}$. The base sequence was determined by Shanghai Biological Technology Co., Ltd. The sequencing results were aligned with Aro I sequence to determine the accuracy of target mutation. The recombinant plasmid with correct sequence was named as pMD18T-simple-Aro I*.

\section{Results and Analysis}

\subsection{Extraction of genomic DNA in Corynebacterium glutamicum LG-332}

The genomic DNA was extracted for agarose gel electrophoresis analysis $(0.8 \%)$. The results showed a higher purity of DNA without RNA contamination. The results were shown in Figure 1:

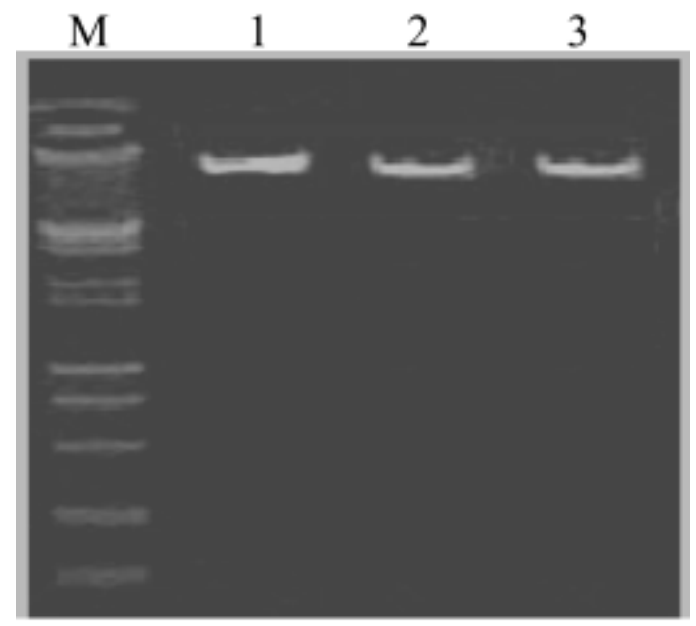

Fig.2 Identification of Genome DNA M: DL-2,000+15,000; 1-3: Genomic DNA

\subsection{PCR amplification of DAHP synthase gene}

The genomic DNA in LG-332 was used as the template to amplify the gene fragment of DAHP synthase (AroD), and the agarose gel electrophoresis (1\%) was shown in Figure 2. The length of the gene fragment was about l.lkb, consistent with the expected theoretical value. 


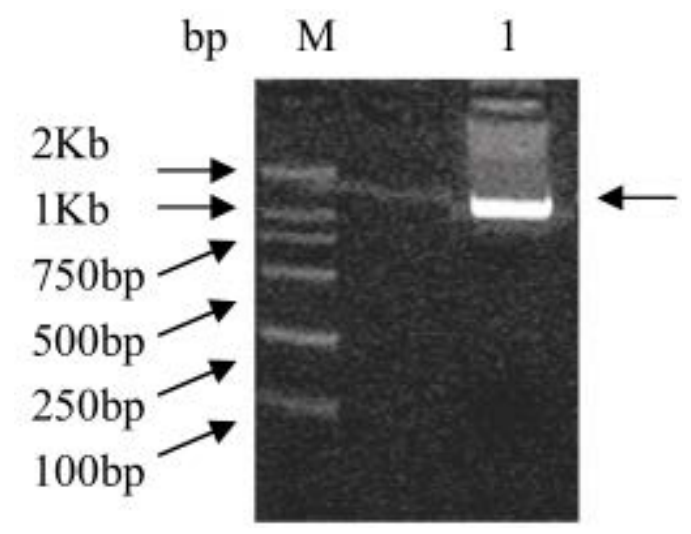

Fig.3 Agrose gel electrophoretic profile of PCR product

M: DL2000; I: Amplified DNA fragment

\subsection{Cloning of DAHP synthase gene and identification of mutant gene}

The purified T-tailed PCR product was ligated to T vector and subcloned. After the identification of bacterium solution and enzyme digestion (Fig. 3), the right clones were sent for sequencing. The sequencing results were analyzed by using DNAMAN software and online BLAST analysis and had $99 \%$ of homology with Aro I sequence logged on GENBANK, indicating a successful gene cloning of DAHP synthase.

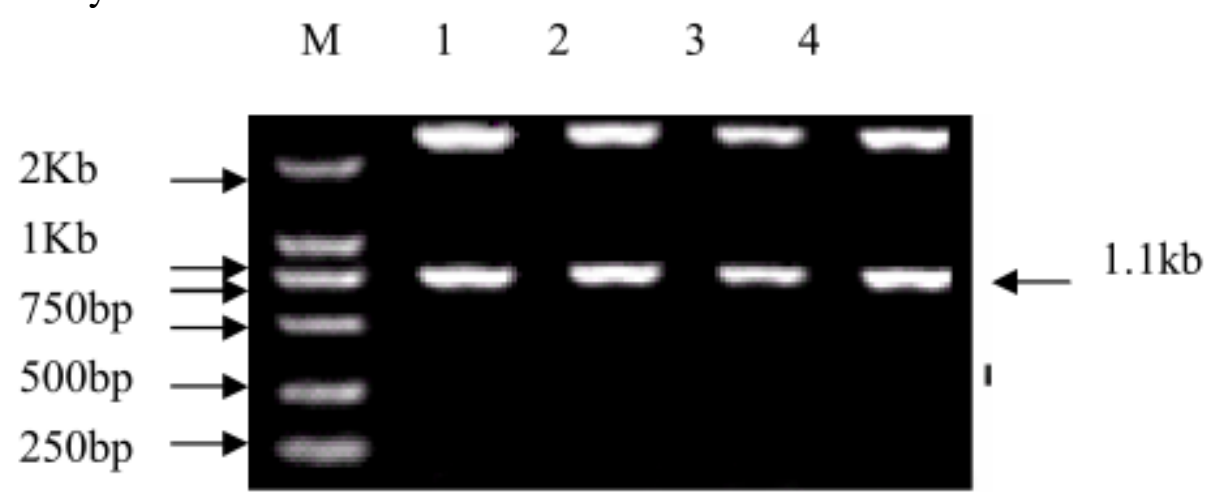

Fig. 4 Identification of restrictive enzyme reaction. M: DL2000; 1-2: restrictive enzyme reaction of pMD18T-simple-Aro I; 3-4 restrictive enzyme reaction of pMD18T-simple-Aro I *

\subsection{Nucleotide sequence alignment of the recombinant plasmid with mutation DS enzyme}

The sequencing results were analyzed by using DNA MAN software and had 99\% homology with Aro I sequence logged on GENBANK, which was completely consistent with the design of mutagenic primers. The nucleotide $\mathrm{T}$ at 607 site was mutated, which indicating a successful gene site-directed mutagenesis of DS enzyme.

\section{Discussion}

In this study, Prime STAR HS polymerase with 3' to 5' exonuclease activity was used to reduce the mismatch rate and improve the accuracy of PCR amplification. With the genomic DNA in high tryptophan-producing Corynebacterium glutamicum LG-332 obtained by multiple screening in this laboratory as the template, the cloned Aro I gene had 100\% homology with the Aro I gene (ATCC13032) logged on GENBANK (GeneID: 1018979). It was not only indicted the cloning fidelity ${ }^{[5,6]}$, but also showed that the double auxotrophy of phenylalanine and tyrosine in LG-332 and the characteristics of resistance to various substrate analogs of tryptophan were not related to the amino acid composition of DAHP enzyme ${ }^{[7]}$.

Combining with cloning fidelity, site-directed mutagenesis of Aro I gene was conducted through one-step mediated method by using simple, rapid and economical polymerase chain reaction with high mutation efficiency. The mutation site was located in the middle of primers. After the temperature cycle, the primers brought the mutation into the newly generated amplification product 
with notched incomplete plasmids. After completion of the entire amplification, Dpn I enzyme was used for digestion of the methylated original template strand. The product was then transformed to competent cells. The ligase could repair the notch in bacteria. Thus the complete plasmid could be generated and normally reproduced in bacteria. After plate culture, more than $90 \%$ of the clones would be with mutations ${ }^{[8]}$.

To verify the catalytic activity of this mutant gene translating into proteins, the future researches on prokaryotic expression of mutant gene and wild gene will be conducted to verify whether the catalytic activity of recombinant enzyme has been improved.

\section{Conclusion}

DAHP synthase gene in Corynebacterium glutamicum (LG-332) was successfully cloned with genomic DNA in Corynebacterium glutamicum (LG-332) as the template, which had a 100\% homology with the DAHP gene sequence logged on GENBANK.

The site-directed mutagenesis was successfully performed on DAHP synthase gene LG-332 in Corynebacterium glutamicum (LG-332) to obtain the L183Q mutant, laying the foundation for the following prokaryotic expression and construction of genetically engineered bacteria.

\section{Acknowledgement}

The authors would like to thank the Specialized Research Fund for the Doctoral Program of Higher Education "Effect of directed evolution to improve DS activity on metabolic flux of tryptophan in Corynebacterium glutamicum (20092223110002)” for the support.

\section{References}

[1] ZHANG Chunhua, ZHAO Zhi, HANG Yingzi, et al. Cloning, expression and sequence analysis of DS I gene in Corynebacterium pekinense AS1.299 and PD-67[J]. Journal of Microbiology. 2008(11): 1466-1472.

[2] HU Yaohui, WANG Yuhua, YU Hansong, et al. Advance of L-tryptophan Application and Production Technology[J]. Journal of Jilin Agricultural University. 2008 (04):586-589.

[3] CraigM E, Wong C H, Alexander J. et al. Delayed referral of new-onset type l diabetes increases the risk of diabetic ketoacidosis[J]. Med J Aust. 2009, 190(4): 219.

[4] J. Sambrook and D.W. Russell. Molecular Cloning: A Laboratory Manual (Translated by HUANG Pei-tang et al). Beijing: Science Press, 2002.

[5] JIANG Pei, SHI Mi, QIAN Zhikang, et al. Secretory expression of Escherichia coli aroG genes in Bacillus subtilis[J]. Journal of Fudan University (Natural Science). 2000(03): 287-291.

[6] Koffas M A, Ramamoorthi R. Pine W A, et al. Sequence of the Corynebacterium glutamicum pyruvate carboxylase gene[J]. Appl Microbiol Biotechnol. 1998, 50(3): 346-352.

[7] LIU Aimnin, GE Haipeng, ZHANG Yuanying, et al. Cloning and expression of aroG gene and series expression of pheA.tyrB gene in E. coli [J]. Acta Biochimica et Biophysica Sinica. 1998(06): 63-66.

[8] Izumi Y, Tatsumi K, Okamoto S. et al. A novel mutation of the KALI gene in Kallmann syndrome[J]. Endocr J. 1999, 46(5): 651-658. 